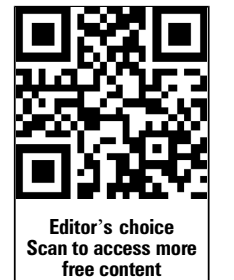

free content

\title{
Obesity accelerates Helicobacter felis-induced gastric carcinogenesis by enhancing immature myeloid cell trafficking and $\mathrm{T}_{\mathrm{H}} 17$ response
}

\author{
Russell E Ericksen, ${ }^{1}$ Shannon Rose, ${ }^{2}$ Christoph Benedikt Westphalen, ${ }^{2}$ \\ Wataru Shibata, ${ }^{3}$ Sureshkumar Muthupalani, ${ }^{4}$ Yagnesh Tailor, ${ }^{2}$ Richard A Friedman, ${ }^{5}$ \\ Weiping Han, ${ }^{1}$ James G Fox, ${ }^{4}$ Anthony W Ferrante $\mathrm{Jr}^{6}{ }^{6}$ Timothy C Wang ${ }^{2}$
}

\section{- Additional material is published online only. To view please visit the journal online (http://dx.doi.org/10.1136/ gutjnl-2013-305092) \\ ${ }^{1}$ Singapore Bioimaging Consortium, Agency for Science, Technology and Research, Singapore, Singapore ${ }^{2}$ Division of Digestive and Liver Diseases, Columbia University, New York, New York, USA \\ ${ }^{3}$ Department of Gastroenterology, Yokohama City University, Yokohama, Japan \\ ${ }^{4}$ Division of Comparative Medicine, Massachusetts Institute of Technology, Cambridge, Massachusetts, USA \\ ${ }^{5}$ Biomedical Informatics Shared Resource, Herbert Irving Comprehensive Cancer Center, Columbia University, New York, New York, USA ${ }^{6}$ Division of Diabetes and Endocrinology, Columbia University, New York, New York, USA}

\section{Correspondence to} Dr Timothy C Wang, Division of Digestive and Liver Diseases, Department of Medicine and Irving Cancer Research Center, Columbia University Medical Center, 1130 Saint Nicholas Avenue, Room \#925, New York, NY 10032-3802, USA:

tcw21@columbia.edu

Received 12 April 2013 Revised 3 May 2013 Accepted 6 May 2013 Published Online First 31 May 2013

\begin{tabular}{l}
\hline To cite: Ericksen RE, \\
Rose S, Westphalen CB, \\
et al. Gut 2014;63: \\
385-394. \\
\hline
\end{tabular}

\section{ABSTRACT \\ Objective To investigate the role of obesity-associated inflammation and immune modulation in gastric carcinogenesis during Helicobacter-induced chronic gastric inflammation.}

Design C57BL/6 male mice were infected with $H$ felis and placed on a high-fat diet (45\% calories from fat). Study animals were analysed for gastric and adipose pathology, inflammatory markers in serum, stomach and adipose tissue, and immune responses in blood, spleen, stomach and adipose tissue.

Results $H$ felis-induced gastric carcinogenesis was accelerated in diet-induced obese mice compared with lean controls. Obesity increased bone marrow-derived immature myeloid cells in blood and gastric tissue of $H$ felis-infected mice. Obesity also led to elevations in CD4 T cells, IL-17A, granulocyte macrophage colonystimulating factor, phosphorylated STAT3 and prosurvival gene expression in gastric tissue of $H$ felis-infected mice. Conversely, in adipose tissue of obese mice, $H$ felis infection increased macrophage accumulation and expression of IL-6, C-C motif ligand 7 (CCL7) and leptin. Finally, the combination of obesity and gastric inflammation synergistically increased serum proinflammatory cytokines, including IL-6.

Conclusions Here, we have established a model to study the molecular mechanism by which obesity predisposes individuals to gastric cancer. In $\mathrm{H}$ felisinfected mice, obesity increased proinflammatory immune responses and accelerated gastric carcinogenesis. Interestingly, gastric inflammation augmented obesity-induced adipose inflammation and production of adipose-derived factors in obese, but not lean, mice. Our findings suggest that obesity accelerates Helicobacter-associated gastric cancer through cytokinemediated cross-talk between inflamed gastric and adipose tissues, augmenting immune responses at both tissue sites, and thereby contributing to a protumorigenic gastric microenvironment.

\section{INTRODUCTION}

Gastric cancer is the fourth most common cancer and the second leading cause of cancer-related death worldwide. ${ }^{1}$ Helicobacter pylori, a bacterium that infects humans and colonises the stomach, causing chronic gastritis and gastric cancer. ${ }^{2-4}$ Among all individuals infected with $H$ pylori, approximately $1-3 \%$ progress to gastric cancer. ${ }^{1}$

\section{Significance of this study}

What is already known on this subject?

- Obesity causes a state of systemic low-grade inflammation and is correlated with increased risk of certain cancers, including gastric cancer.

- Adipose-derived factors can promote tumour cell growth in vivo and in vitro.

- In humans, serum IL-6 concentration is elevated in obese individuals and is positively correlated with gastric cancer development and progression.

\section{What are the new findings?}

- Diet-induced obesity accelerated Helicobacter felis-induced gastric inflammation and carcinogenesis.

- Obesity promoted a protumorigenic gastric microenvironment and induced gastric STAT3 signalling.

- Obesity enhanced IMC recruitment and $\mathrm{T}_{\mathrm{H}}$ 17-associated responses in the stomachs of Helicobacter-infected mice.

- High-fat diet plus Helicobacter felis infection significantly increased serum IL-6 and leptin concentrations, compared with obesity or infection alone.

- $H$ felis infection increased macrophage accumulation, as well as CCL7 and leptin expression, in obese, but not lean, visceral adipose tissue.

As nearly half the world's population is infected, $H$ pylori contributes significantly to the global gastric cancer burden. ${ }^{1}$

Obese individuals not only have a greater risk of certain cancers, including gastric cancer, but also suffer from higher cancer mortality rates. ${ }^{5}$ The incidence of obesity and obesity-associated diseases are rising at an alarming rate worldwide. ${ }^{67}$ Research on these diseases, including type II diabetes and atherosclerosis, highlights obesity-induced inflammation as a common underlying feature. ${ }^{6-9}$ Studies in mice have shown that obesity can promote multiple cancers (including colorectal and pancreatic cancer) and 
Significance of this study

\section{How might it impact on clinical practice in the foreseeable future?}

- Obesity and associated adipose inflammation significantly enhanced $H$ felis-driven gastric carcinogenesis. Modulation of adipose inflammation may improve gastric cancer risk in obese patients. Obesity prevention measures, especially in children, will also play an important role in addressing this cancer risk.

- Serum IL-6 was the most significantly elevated cytokine in obese infected mice. Thus, serum IL-6 levels may be a useful indicator of obese individuals at higher risk of developing gastric cancer.

- Helicobacter infection augmented adipose tissue macrophage infiltration and inflammation in obese mice. Eradication of gastric Helicobacter species infection may improve obesity-associated adipose inflammation and obesity-associated pathologies.

implicates systemic inflammation in this process. ${ }^{10}{ }^{11}$ Research in this field has largely focused on the direct effect of adipose-derived factors on established tumours. However, little is known about the effect of obesity on immune modulation, the establishment of a protumorigenic microenvironment and cancer initiation. ${ }^{7}$

To investigate the effects of obesity on gastric inflammation and carcinogenesis, we placed Helicobacter felis-infected mice on a high-fat diet (HFD, 45\% calories from fat). Chronic administration of a HFD is a clinically relevant model of obesity. ${ }^{12}$ Mice exposed to this diet develop a metabolic phenotype similar to that observed in obese humans, characterised by adipose inflammation, hyperglycaemia and insulin resistance. ${ }^{9} 13$ $H$ felis infection is commonly used as a murine model of gastric cancer, as $H$ felis infection in mice recapitulates the pathological progression from gastritis to dysplasia to cancer observed in chronic human $H$ pylori infection. ${ }^{14}$

Helicobacter-dependent gastric cancer in mice and humans is associated with a sustained gastric immune response that involves cells of both lymphoid and myeloid origin. The circulating and gastric populations of CD11bGr1 immature myeloid cells (IMCs) increase early in the disease process, and have been implicated in cancer initiation. ${ }^{15}$ IMCs are a heterogeneous cell population and include myeloid-derived suppressor cells (MDSCs), among other cell populations. ${ }^{16}$ MDSCs are characterised by their ability to suppress cytotoxic CD8 T cell activation, preventing antitumour immunity and promoting the survival of transformed epithelial cells. ${ }^{16-20}$ Cytokines and growth factors, including interleukin (IL)-6 and granulocyte macrophage-colony stimulating factor (GM-CSF), have been shown to play an important role in MDSC recruitment and activity. ${ }^{16}{ }_{17}$ In addition to IMC involvement, Helicobacter-driven carcinogenesis is also associated with increased $T$ helper $1\left(T_{H} 1\right)$ and $T_{H} 17$ immune responses. ${ }^{21} 22$ The role of $\mathrm{T}_{\mathrm{H}} 17$ cells, and the prototypical $\mathrm{T}_{\mathrm{H}} 17$ cytokine IL-17A, ${ }^{23}$ in tumour development remains controversial and is likely dependent on tissue type, tumour stage and microenvironment. ${ }^{24}{ }^{25}$ Nevertheless, a heightened $\mathrm{T}_{\mathrm{H}} 17$ response has been positively correlated with more advanced gastric pathology, ${ }^{26}$ and attenuation of the $T_{H} 17$ response has been associated with reduced gastric dysplasia. ${ }^{27}$
As in gastric cancer, proinflammatory immune responses are also initiated during obesity. ${ }^{6}$ During obesity, adipose tissue undergoes substantial tissue remodelling as adipocytes become hypertrophic and hyperplastic, causing increased adipose tissue inflammation. ${ }^{6}$ Accumulation of adipose tissue macrophages has been identified as one of the main sources of this adipose inflammation. ${ }^{68}$ Communication between macrophages and adipocytes enhances cytokine production, including tumour necrosis factor (TNF)- $\alpha$, IL-6 and IL-1 $\beta$, which furthers macrophage recruitment, and perpetuates the inflammatory response. ${ }^{8}$ Adipose-derived factors, specifically TNF- $\alpha$, IL- 6 , plasminogen activator inhibitor-1 (PAI-1) and leptin, have been proposed to increase susceptibility to multiple cancers during obesity, but the specific mechanisms are not well understood. ${ }^{12}$ Activation of signal transducer and activator of transcription 3 (STAT3), a known oncoprotein by IL-6 and leptin, has been proposed as one key mechanism involved in obesity-associated cancer risk. ${ }^{12}$ Additionally, high leptin levels have been shown to promote proinflammatory T cells while suppressing regulatory T cells. ${ }^{28}$

The aim of this study was to investigate the effect of diet-induced obesity on Helicobacter-associated gastric inflammation and carcinogenesis. We hypothesised that obesity-induced inflammation and immune activation in adipose tissue would amplify tumorigenic gastric inflammation. We also sought to better define the interaction between immune responses initiated by obesity and gastric helicobacter infection. Our data demonstrate that obesity-associated adipose inflammation enhanced gastric inflammation during $H$ felis infection and accelerated gastric preneoplasia.

\section{MATERIALS AND METHODS}

Male C57BL/6 mice were infected with $H$ felis at 8 weeks as previously described. ${ }^{29}$ Infected and uninfected mice were fed either a HFD (45\% of calories from fat, Research Diets 12451) or a standard chow diet (CD, 13\% of calories from fat, PicoLab Rodent Diet 20) ad libitum. HFD was begun 3 weeks postinfection (PI). Methods for metabolic profile assays, immunohistochemistry (IHC), protein quantification, real-time qRT-PCR, chemokine infusion studies, BrdU injection, flow cytometry, in vitro $\mathrm{T}$ cell differentiation and transwell migration assays are provided in the online supplement.

\section{RESULTS}

\section{Diet-induced obesity accelerates $\boldsymbol{H}$ felis-induced carcinogenesis}

To determine if obesity can augment $H$ felis-driven carcinogenesis, infected mice were fed a HFD ( $45 \%$ calories from fat) or standard CD (13\% calories from fat) from 3 weeks PI until euthanasia at 10 and 15 months PI. Uninfected control mice were placed on the same feeding regimen. Hereafter, we will refer to these four study groups as: $H$ felis/HFD, $H$ felis/CD, uninfected/HFD and uninfected/CD. As expected, mice on HFD gained significantly more weight than mice on $\mathrm{CD}$ (figure $1 \mathrm{~A}$ ). $H$ felis infection did not alter weight gain, body composition, or fasting glucose and insulin levels compared with controls (figure $1 \mathrm{~A}$, see online supplementary figure $\mathrm{S} 1 \mathrm{~A}, \mathrm{~B}$, data not shown). Diet did not alter $H$ felis colonisation density (data not shown).

$H$ felis infection resulted in gastric dysplasia in all animals. Gross gastric lesions in $\mathrm{H}$ felis/HFD mice were roughly twice

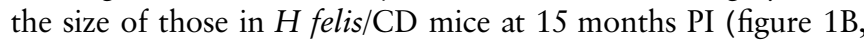
C). In infected mice, obesity significantly increased severity of inflammation, epithelial defects, metaplasia and dysplasia at 


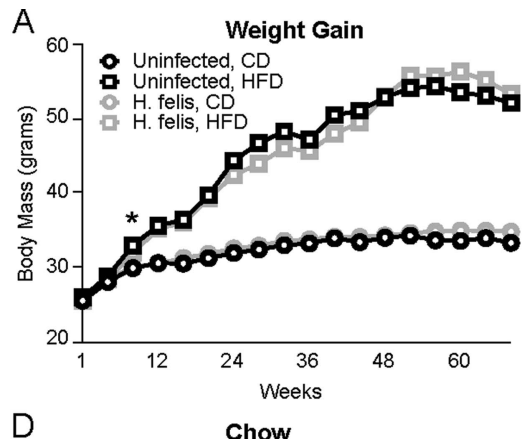

B

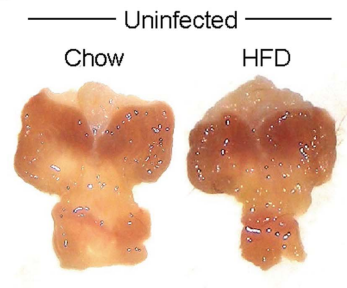

D
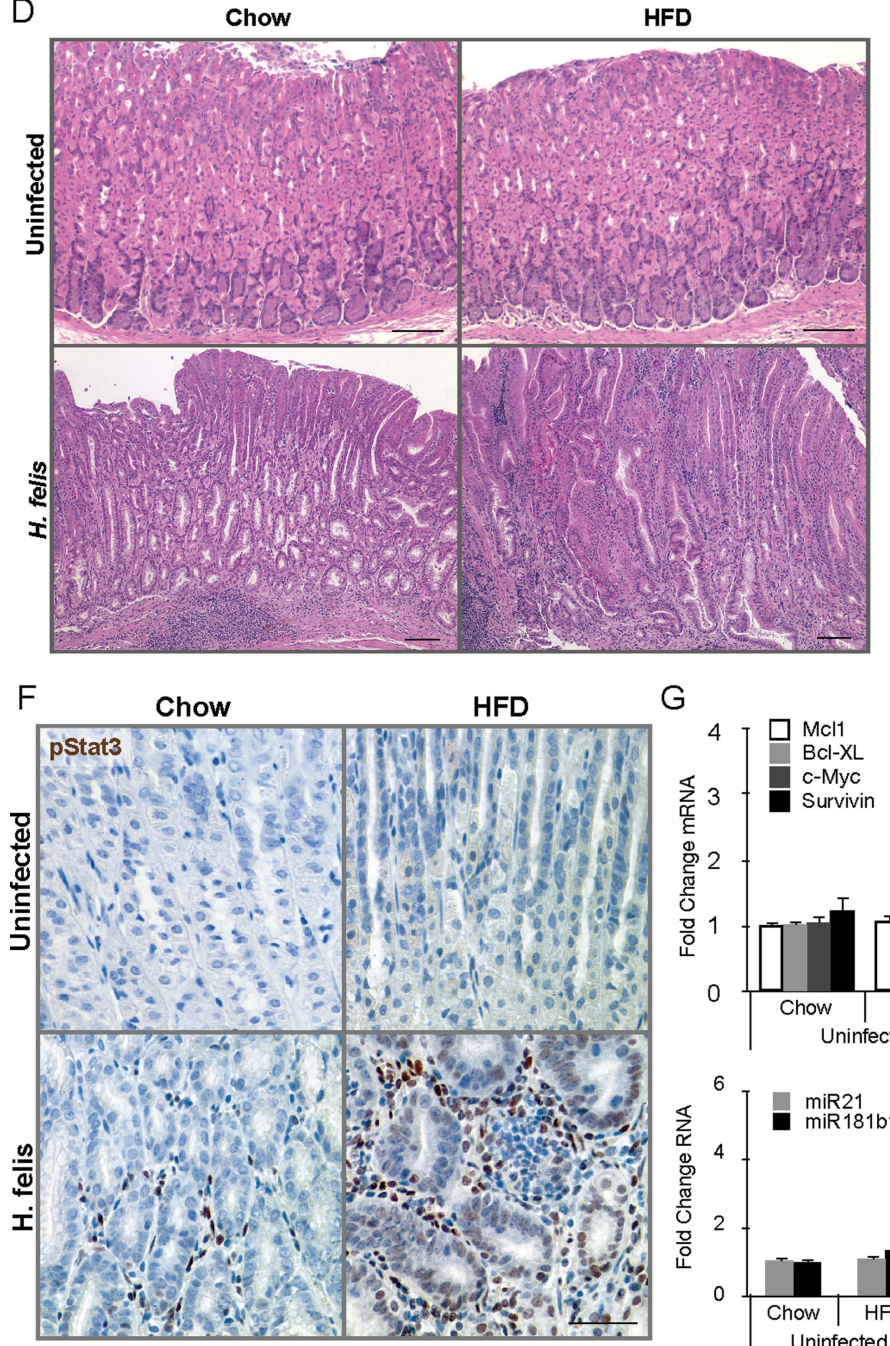

G
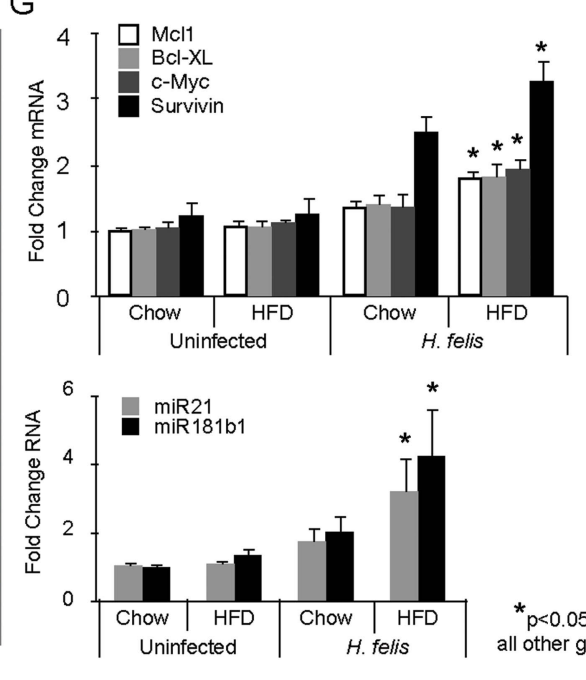

C
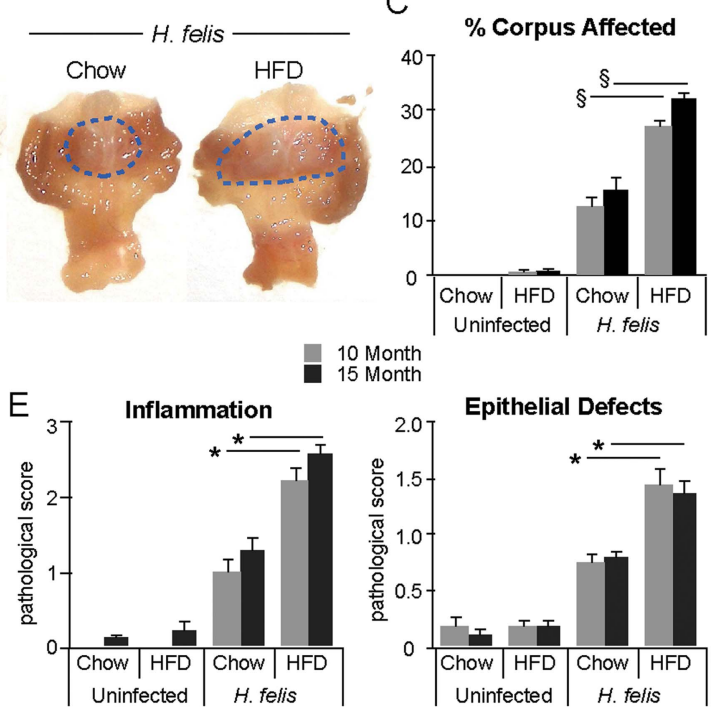

15 Month
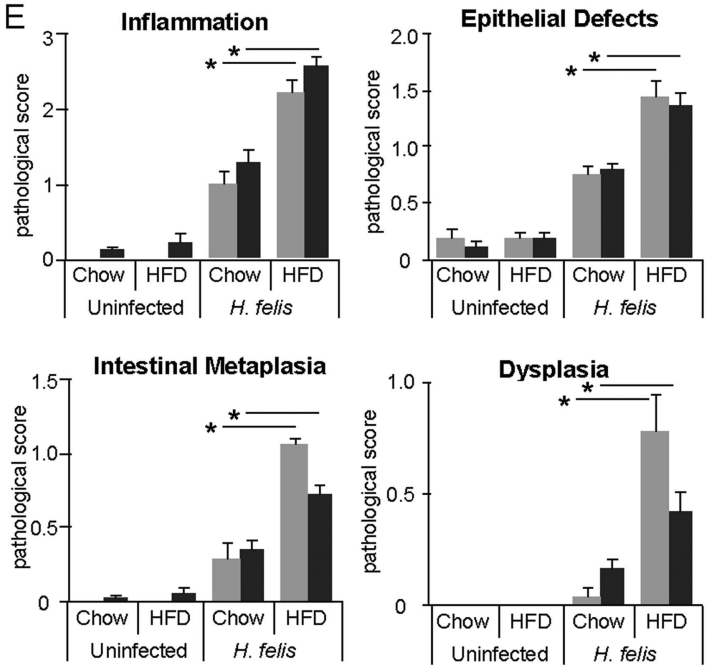

$\mathrm{H}$
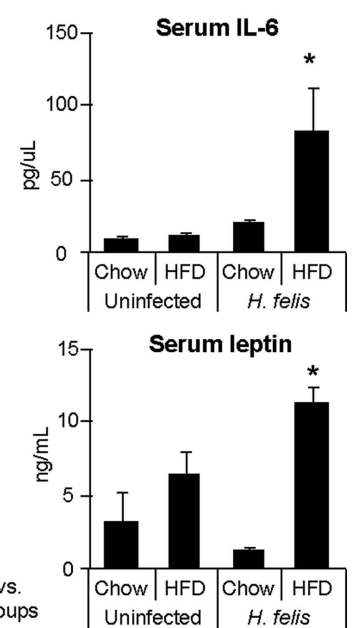

Figure 1 (A) Average body mass ( $\mathrm{g}$ ) of study mice from 1-68 weeks. (B) Representative gross images of stomach, 15 months postinfection (PI). Inflamed/dysplastic region circled by blue dotted line. (C) Percent corpus inflamed/dysplastic, calculated from gross gastric images. (D) Representative images of gastric corpus H\&E histology, 15 months PI. scale bars=100 $\mu \mathrm{m}$. (E) Gastric histopathological scores at 10 and 15 months PI. (F) Representative images of phosphorylated (p)STAT3 immunohistochemistry in gastric corpus. Scale bar=50 $\mu \mathrm{m}$. (G) mRNA quantification of Mcl-1, Bcl-XL, c-Myc, survivin, miR21 and miR181b in gastric corpus by qRT-PCR. (H) Serum IL-6 and leptin protein quantification by milliplex. ${ }^{*} p<0.05$, ${ }^{\#} \mathrm{p}<0.01,{ }^{\S} \mathrm{p}<0.001$.

both time points (figure 1D,E). HFD alone did not induce gastric inflammation or dysplasia (figure 1C-E).

\section{Adipose-derived factors activate STAT3 in gastric tissue}

Sustained STAT3 signalling in epithelial cells has been shown to promote cell survival and proliferation in multiple cancer models, including gastric cancer. ${ }^{25}{ }^{30}$ Accordingly, we analysed gastric corpus tissue for activated, phosphorylated STAT3 (pSTAT3) by IHC. At 15 months PI, $H$ felis/HFD mice had widespread pSTAT3 staining in epithelial and stromal cells compared with controls. $\mathrm{H}$ felis infection alone resulted in moderate stromal and minimal epithelial STAT3 activation. By contrast, $H$ felis plus HFD resulted in widespread stromal and epithelial STAT3 activation, suggesting that HFD may contribute to gastric epithelial cell survival during chronic $H$ felis infection. pSTAT3 staining was extremely rare in uninfected mice (figure 1F). STAT3 can exert oncogenic effects in epithelial cells through upregulation of prosurvival/antiapoptotic genes, including survivin, Bcl-XL, Mcl1 and c-Myc, ${ }^{30}$ and by driving expression of certain microRNAs, such as miR21 and miR181b1. ${ }^{31}$ 
A

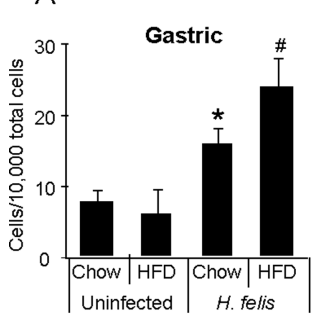

C
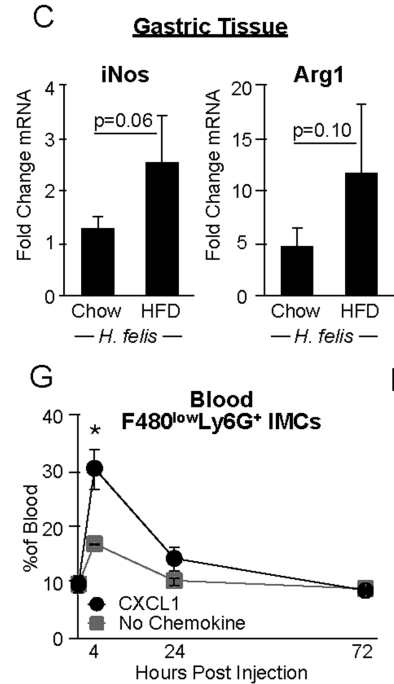

Gr1+/CD11b+ IMC

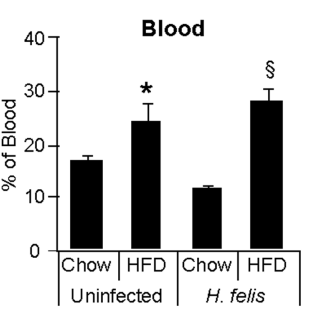

D

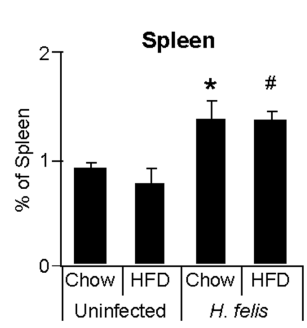

E
B

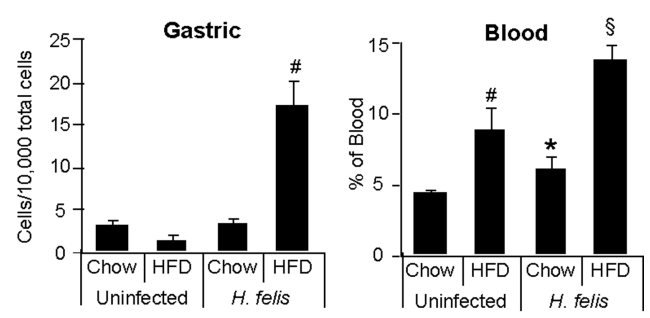

Figure 2 ( $A$ and $B$ ) Cells isolated from stomach, blood and spleen, stained with anti-CD45, CD11b, Gr1, F4/80, Ly6C and Ly6G antibodies, analysed by flow cytometry. (A) Total Gr1CD11b immature myeloid cell (IMC) quantification in gastric tissue, blood and spleen. (B) F4/80 ${ }^{\text {low }}$ Ly6G IMC subset quantification in gastric tissue and blood. (C) Arg1 and iNOS mRNA quantification from whole corpus gastric tissue by qRT-PCR. (D) Serum CXCL1 protein quantification by milliplex. (E) CXCL1 mRNA quantification in gastric corpus and visceral adipose by qRT-PCR. $p$ Values versus uninfected/CD. (F) Transwell migration assay using fluorescence-activated cell sorting sorted CD11bGr1 IMCs from uninfected/CD whole bone marrow. Migrated cells stained with anti-F4/80, anti-Ly6C and anti-Ly6G antibodies, quantified with counting beads by flow cytometry; $n=3-4$ wells/condition, one representative shown of three independent experiments, $p$ values versus uninfected/CD. (G and $H$ ) Intravenous injection of recombinant murine CXCL1 (40 $\mu \mathrm{g} / \mathrm{kg}$ body mass) or sterile PBS. Blood (G) and bone marrow $(\mathrm{H})$ cells stained with anti-CD45, CD11b, Gr1, F4/80, Ly6C and Ly6G antibodies, quantified by flow cytometry; $n=3-4$ mice/condition. ${ }^{*} p<0.05,{ }^{\#} p<0.01,{ }^{\S} p<0.001$. CXCL, Chemokine $C-X-C$ motif ligand.

Expression of these STAT3 target genes and microRNAs were significantly upregulated in gastric tissues of $\mathrm{H}$ felis/HFD mice compared with $\mathrm{H}$ felis/CD mice (figure $1 \mathrm{G}$ ). To identify possible initiators of the observed gastric STAT3 activity, we assessed factors known to initiate STAT3 phosphorylation and nuclear translocation, including IL-6, IL-11 and leptin. ${ }^{5} 30$ Interestingly, HFD did not increase expression of IL-6 or IL-11 in gastric corpus tissue of infected mice (see online supplementary figure S1C). It is possible that these factors were upregulated in other tissues, such as adipose tissue, and travelled to the stomach to activate STAT3, therefore, we measured IL- 6 and leptin concentrations in the serum. We detected increased serum concentration of IL-6 and leptin in $H$ felis/HFD mice, compared with $H$ felis/CD mice (figure $1 \mathrm{H}$ ). These data suggest that obesity and $H$ felis infection synergistically increased serum IL-6 and leptin levels, likely enhancing STAT3-mediated gastric epithelial transformation beyond that observed with $\mathrm{H}$ felis infection alone.

\section{Obesity enhances IMC trafficking}

During obesity, bone marrow-derived IMCs traffic to adipose tissue, but it is unknown if these populations interact or overlap with IMCs implicated in gastric tumorigenesis. ${ }^{32}$ Using flow cytometry, we quantified CD11bGR1 IMC subpopulations in the stomach, blood and spleen, using the cell surface markers
F4/80, Ly6C and Ly6G (for gating strategy, see online supplementary figure S2A). CD11bGR1 IMCs were significantly elevated in gastric and splenic tissues of all infected mice, and in the blood of all mice on HFD (figure 2A). In gastric tissue and blood, F4/80 ${ }^{\text {low } L y 6 G ~ I M C s ~ w e r e ~ s i g n i f i c a n t l y ~ e l e v a t e d ~ i n ~}$ $H$ felis/HFD mice compared with $H$ felis/CD mice, indicating that this neutrophil-like IMC subset ${ }^{4}$ is most sensitive to a HFD in the context of $\mathrm{H}$ felis infection (figure 2B). Additional IMC populations were modulated by $\mathrm{H}$ felis or HFD status, though blood or spleen concentrations did not correlate with gastric levels (see online supplementary figure S2B).

MDSCs, a subset of CD11bGR1 IMCs, can curb proliferation of cytotoxic CD8 T cells and dampen antitumour immunity, partially through the production of arginase $1(\operatorname{Arg} 1)$ and inducible nitric oxide synthase (iNOS). ${ }^{3}$ A trend toward increased gastric Arg1 and iNOS expression was observed in $H$ felis/HFD mice compared with $H$ felis/CD mice, suggesting that IMCs recruited to the stomach of $\mathrm{H}$ felis/HFD mice exhibit immunosuppressive activity (figure $2 \mathrm{C}$ ). Collectively, these data indicate that obesity may accelerate $H$ felis-dependent carcinogenesis by increasing MDSC accumulation in the stomach.

To identify chemokines involved in IMC recruitment, we measured serum levels of chemokine C-X-C motif ligand (CXCL)- 1, 2, 5 and C-C motif ligand (CCL)- 2, 3, 4. Of these, CXCL1 was the only chemokine significantly elevated in serum 


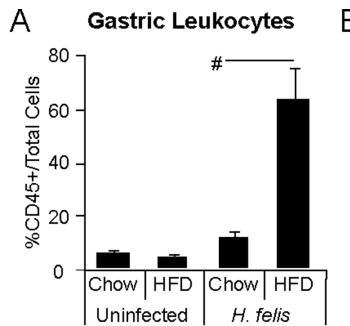

C

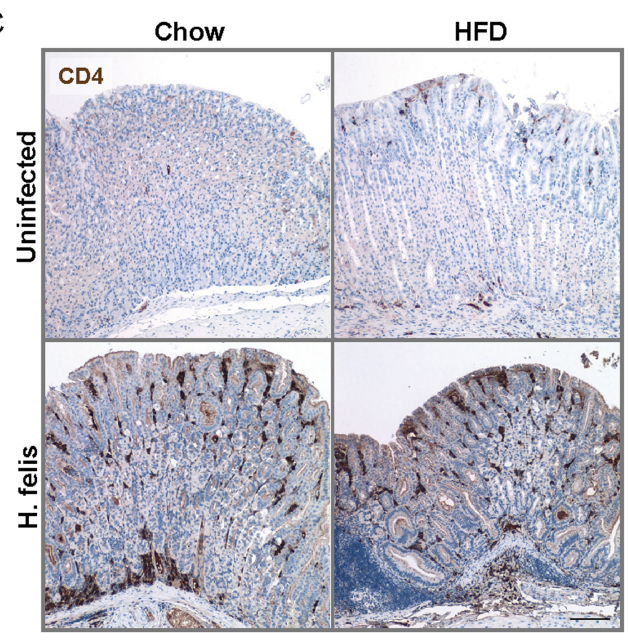

\section{B Gastric T cells}
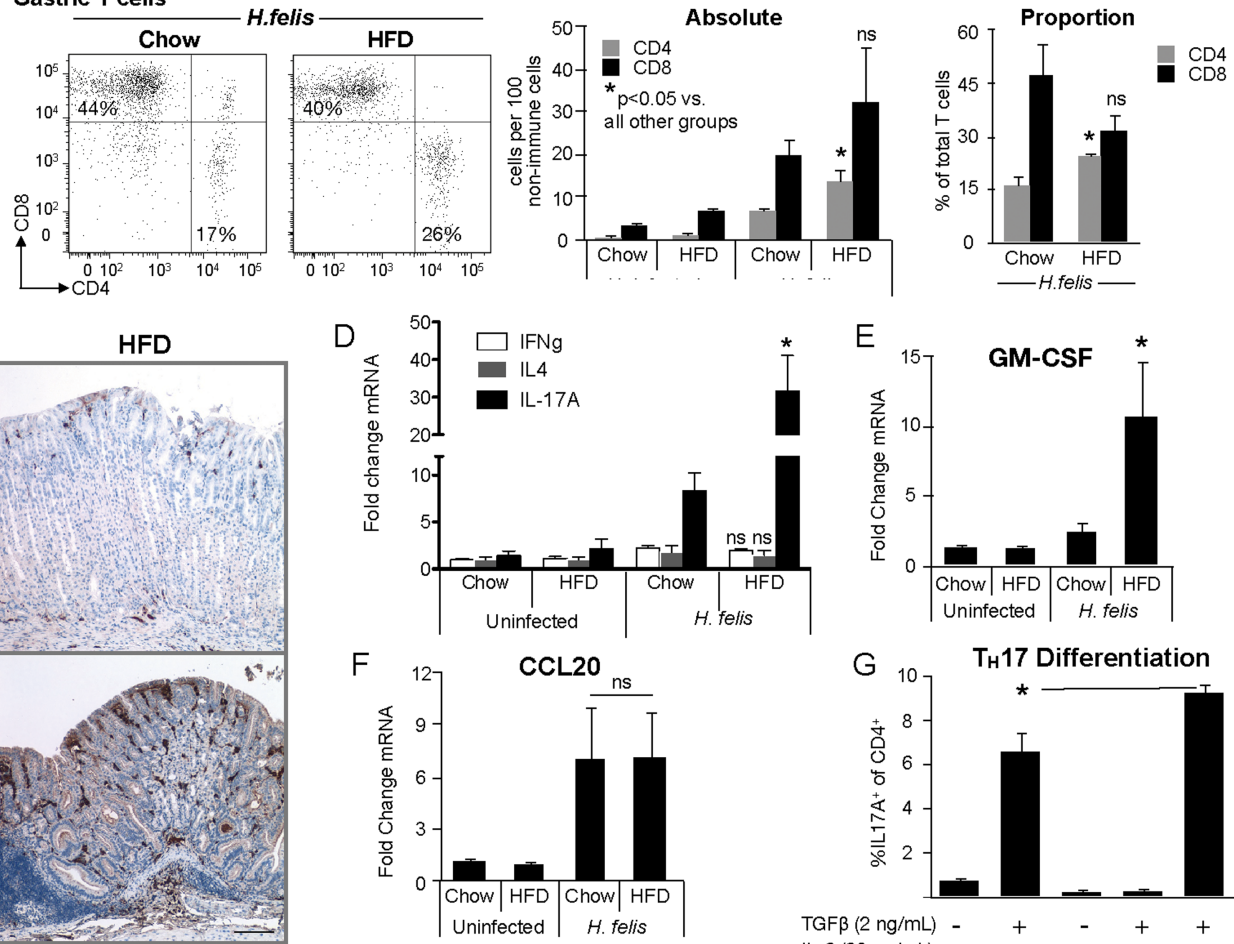

E
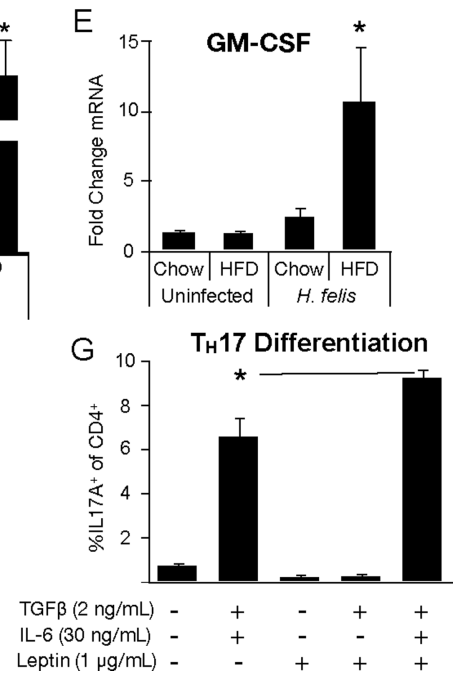

Figure 3 (A) Gastric leukocytes isolated from whole gastric tissue, stained with anti-CD45, CD3, CD4 and CD8 antibodies, quantified by flow cytometry. CD45 gastric leukocytes normalised to total isolated cells. (B) Representative plots for T cell CD4 and CD8 expression, pregated for CD45CD3 cells. Absolute CD4 and CD8 T cells, normalised to 100 CD45 non-immune cells. Proportional gastric CD4 T cells and CD8 T cells, normalised to total T cells (CD45CD3). (C) Representative images of CD4 immunohistochemistry in gastric corpus. Scale bar=200 $\mu \mathrm{m}$. (D-F) mRNA quantification of IL-4, and IFN $\gamma$ and IL-17A (D), GM-CSF (E) and CCL20 (F) in gastric corpus by qRT-PCR. (G) In vitro $T_{H} 17$ differentiation from isolated naive T cells, analysed by flow cytometry for intracellular IL-17A. N=3-4 wells/condition, one representative of three independent experiments shown. ${ }^{*} \mathrm{p}<0.05,{ }^{\#} \mathrm{p}<0.01,{ }^{\S} \mathrm{p}<0.001$. CCL, C-C motif ligand; GM-CSF, granulocyte macrophage-colony stimulating factor; IFN, interferon.

of $\mathrm{H}$ felis/HFD mice compared with all control groups (figure $2 \mathrm{D}$, data not shown). This finding is consistent with data reporting a role for CXCL1 in the recruitment of neutrophil-like IMCs. ${ }^{33}{ }^{34}$ To identify the possible sources of serum CXCL1, we quantified adipose and gastric CXCL1 mRNA. In adipose tissue, CXCL1 expression was increased almost sixfold in $H$ felis/HFD compared with $H$ felis/CD. In gastric tissue, CXCL1 expression was increased threefold in $H$ felis/HFD compared with $H$ felis/CD mice. We observed no synergistic upregulation of CXCL1 in adipose or gastric tissue of $\mathrm{H}$ felis/HFD mice (figure 2E). Thus, it appears that HFD and $H$ felis infection independently upregulate CXCL1 expression in the adipose tissue and stomach, respectively. The elevated serum CXCL1 protein observed in $\mathrm{H}$ felis/HFD mice is likely a result of increased CXCL1 secretion from both gastric and adipose tissues, although adipose tissue may be a larger contributor. To test if CXCL1 can directly affect F4/80 ${ }^{\text {low }}$ Ly6G IMC mobilisation, we performed a transwell migration assay using CD11bGr1 IMCs isolated from bone marrow of uninfected lean mice. Indeed, CXCL1 caused a potent and dose-dependent migratory response of $\mathrm{F} 4 / 80^{\mathrm{low}} \mathrm{Ly} 6 \mathrm{G}$ IMCs (figure $2 \mathrm{~F}$ ). To demonstrate the specificity of the migration assay, we applied F4/ $80^{\text {int }}$ Ly6C IMCs to the transwell, and confirmed that this monocyte-like subpopulation did not migrate toward CXCL1 (see online supplementary figure S2C). Additionally, while F4/80 $0^{\text {int }}$ Ly6C IMCs did respond to monocytic chemokines CCL2 and CCL7, F4/80 ${ }^{\text {low }}$ Ly6G IMCs did not (figure 2 F, see online supplementary figure S2C). To corroborate these finding in vivo, we performed intravenous infusions of CXCL1 $(40 \mu \mathrm{g} /$ $\mathrm{kg}$ body weight). Four hours after CXCL1 injection, F4/ $80^{\text {low }}$ Ly6G IMCs in the blood increased threefold and returned to baseline at $24 \mathrm{~h}$ (figure 2G). Concomitantly, bone marrow F4/80 ${ }^{\text {low }}$ Ly6G IMCs decreased sharply $4 \mathrm{~h}$ postinjection, and returned to baseline at $24 \mathrm{~h}$ (figure $2 \mathrm{H}$ ). No changes in IMC mobilisation were observed in control mice infused with phosphate-buffered saline (PBS) alone (data not shown). Taken together, these results suggest that increased serum CXCL1, largely due to increased adipose CXCL1 expression in obesity, may facilitate $\mathrm{F} 4 / 80^{\mathrm{low}} \mathrm{Ly} 6 \mathrm{G}$ IMC trafficking from bone marrow to infected gastric tissue. Furthermore, increased F4/80 ${ }^{\text {low }} \mathrm{Ly} 6 \mathrm{G}$ IMC mobilisation was correlated with accelerated progression of carcinogenesis in obese infected mice, suggesting involvement of this IMC subpopulation in the disease process.

\section{Obesity enhances gastric $T_{H} 17$ response in $\boldsymbol{H}$ felis-infected mice}

Infiltrating immune cells, particularly $\mathrm{T}$ cells, are central to Helicobacter-induced gastritis and cancer. ${ }^{2}{ }^{21}$ Therefore, we began by characterising immune cell populations in gastric tissue of our study mice. With addition of HFD, we saw a large influx of CD45 leukocytes in the stomach of $H$ felis-infected mice, compared with lean infected controls (figure $3 \mathrm{~A}$ ). In $\mathrm{H}$ felis/HFD mice, there was a significant increase in CD4 Tcells and a modest non-significant decrease in CD8 T cells, compared with $\mathrm{H}$ felis/ $\mathrm{CD}$ mice (figure 3B). CD4 IHC confirmed that CD4 cells were heavily recruited during infection, particularly with HFD. With 
A

B

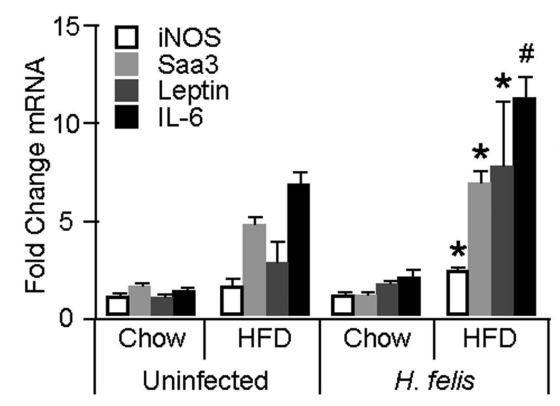

C

SVF Concentration
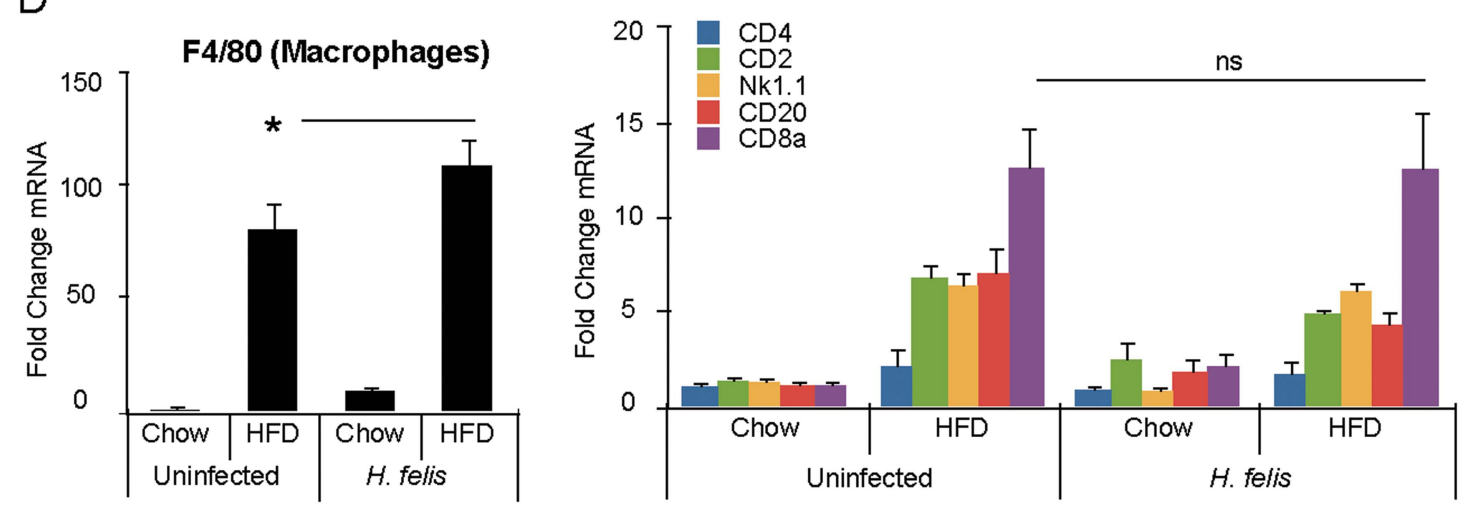

E

H. felis

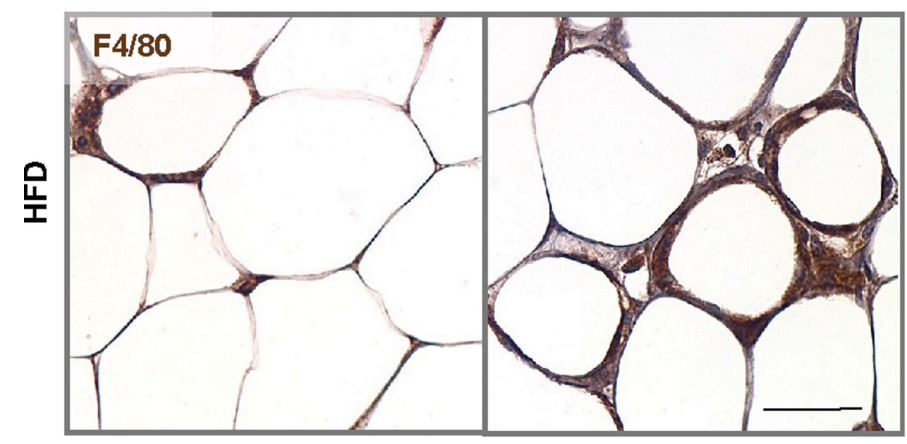

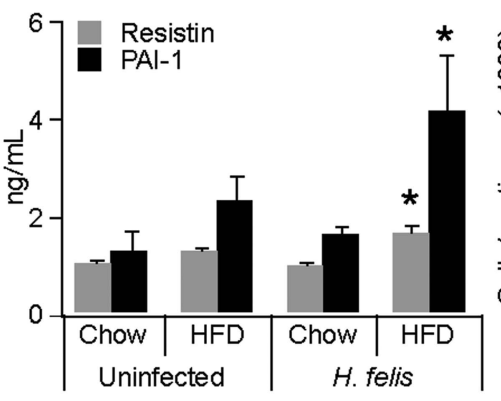

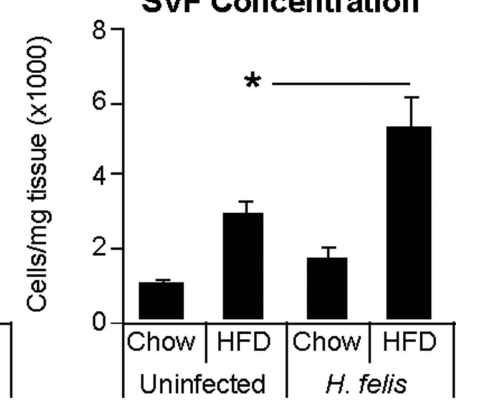

Uninfected $H$. felis

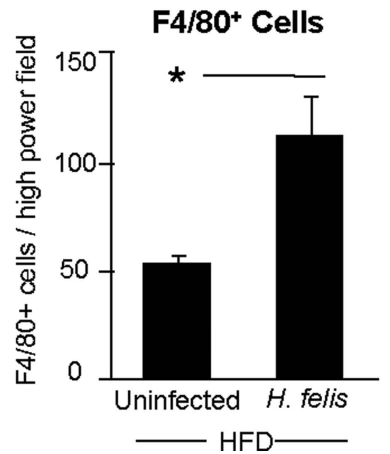

Figure 4 (A) mRNA quantification of iNOS, Saa3, leptin and IL-6 in visceral adipose by qRT-PCR. (B) Serum PAI-1 and resistin protein quantification by milliplex. (C) Stromal vascular section isolated from visceral adipose, quantified by haemocytometer, normalised to tissue weight. (D) mRNA quantification of F4/80, CD4, CD2, NK1.1, CD20 and CD8 $\alpha$ in visceral adipose by qRT-PCR. (E) Representative images of F4/80 immunohistochemistry in visceral adipose of high-fat diet groups. Minimum 10 representative fields quantified per mouse. Scale bar $=50 \mu \mathrm{m}$. ${ }^{*} p<0.05,{ }^{\#} p<0.01,{ }^{\S} p<0.001$.

$H$ felis infection, CD4 cells were scattered throughout the epithelium, though they primarily aggregated at the base or top of glands, and were rarely found within lymphoid aggregates (figure 3C).

Cells expressing the CD4 surface antigen include the $\mathrm{T}_{\mathrm{H}}$ cell populations $\mathrm{T}_{\mathrm{H}} 1, \mathrm{~T}_{\mathrm{H}} 2$ and $\mathrm{T}_{\mathrm{H}} 17 .{ }^{23}{ }^{30}$ Gastric tissues were screened for changes in expression of cytokines classically associated with these $\mathrm{T}_{\mathrm{H}}$ cell populations (figure $3 \mathrm{D}$ ). We observed increased IL-17A, suggesting a heightened $\mathrm{T}_{\mathrm{H}} 17$ response (figure 3D). Interferon (IFN) $\gamma$ and IL-4 expression were only modestly elevated upon administration of HFD to infected mice (figure $3 \mathrm{D}$ ), suggesting that the $\mathrm{T}_{\mathrm{H}} 1$ and $\mathrm{T}_{\mathrm{H}} 2$ responses are minimally involved. Elevated gastric IL-17A protein was confirmed by ELISA and IHC (see online supplementary figure S3A,B). Furthermore, gastric GM-CSF expression was increased in $\mathrm{H}$ felis/HFD mice compared with $\mathrm{H}$ felis/CD mice (figure $3 \mathrm{E}$ ), which has been strongly linked to $\mathrm{T}_{\mathrm{H}} 17$ pathogenesis. $^{24}$ Expression of other $\mathrm{T}_{\mathrm{H}} 17$-associated cytokines (IL-17F, IL-21, IL-22, IL-6 and IL-1 $\beta$ ) were increased with $H$ felis infection but unaffected by diet (see online supplementary figures S1C,S3C). IL-11 has been shown to induce gastric tumours in genetic models, ${ }^{35}$ however, we observed no change in gastric IL-11 expression (see online supplementary figure S1C).

To assess changes in $\mathrm{T}_{\mathrm{H}} 17$ recruitment we measured gastric expression of CCL20, a chemokine that mediates $\mathrm{T}_{\mathrm{H}} 17$ cell trafficking. ${ }^{36}$ CCL20 mRNA expression was unaffected by diet in infected mice, so it is unlikely that HFD influences $T_{H} 17$ recruitment (figure $3 \mathrm{~F}$ ). Next we investigated changes in $\mathrm{T}_{\mathrm{H}} 17$ differentiation. Cytokines involved in $\mathrm{T}_{\mathrm{H}} 17$ differentiation and maintenance, including IL-6, IL-1 $\beta$ and IL-21, were not elevated in gastric tissue of $\mathrm{H}$ felis/HFD mice compared with $\mathrm{H}$ felis/ chow mice (see online supplementary figure S3C). Therefore, 
we speculate that circulating $\mathrm{T}_{\mathrm{H}}$ 17-differentiation factors, including IL-6, are responsible for enhanced $\mathrm{T}_{\mathrm{H}} 17$ development. In vitro studies have shown that IL- 6 and TGF- $\beta$ together are sufficient to generate IL-17A-producing $\mathrm{T}_{\mathrm{H}} 17$ cells from isolated naïve $\mathrm{T}$ cells. ${ }^{2}{ }^{30}$ Interestingly, using this in vitro assay, we found that leptin increased the percentage of IL-17A-expressing T cells in the presence of IL- 6 and TGF- $\beta$, but had no significant effect alone (figure $3 \mathrm{G}$ ). These data suggest that together elevated serum IL- 6 and leptin in obese infected mice contributed to a pathogenic $\mathrm{T}_{\mathrm{H}} 17$ response in the stomach.

\section{$H$ felis infection increases adipose inflammation in obese mice}

Next, we investigated the effect of $H$ felis infection on adipose inflammation. In obese mice, $H$ felis infection significantly increased visceral adipose IL-6, leptin, Saa3 and iNOS expression (figure 4A). These factors have proinflammatory activity and are elevated in adipose tissue of obese humans. ${ }^{3} 928$ Not all proinflammatory cytokines associated with obesity were upregulated by $H$ felis infection, as TNF- $\alpha$ and IL- $1 \beta$ expression were similar in obese and lean infected groups (see online supplementary figure S4A). We also observed a significant increase of two classical adipose-derived inflammatory factors, PAI-1 and resistin ${ }^{24} 37$ in the serum of $H$ felis/HFD mice compared with uninfected/HFD controls (figure 4B). Adipose tissue contains many cell populations in addition to adipocytes, including resident immune cells, which are found in the adipose stromal vascular fraction (SVF). Thus, adipose SVF density can provide a measure of immune cell accumulation. ${ }^{12}$ Indeed, adipose SVF cells were increased in $H$ felis/HFD mice was significantly increased compared with uninfected/HFD mice (figure 4C). This finding was confirmed by calculation of nuclei density in visceral adipose tissue $\mathrm{H} \& \mathrm{E}$ staining (see online supplementary figure S4B). Interestingly, $\mathrm{H}$ felis infection did not induce adipose inflammation in lean mice for any parameter tested (figure 4, see online supplementary figure S4). Stromal cell accumulation has been reported to correlate with both adipocyte size and overall body adiposity, ${ }^{6}{ }^{8}$ but we observed no difference in these parameters between HFD groups (figure 4C, see online supplementary figures $\mathrm{S} 1 \mathrm{~B}, \mathrm{~S} 4 \mathrm{~B}$ ).

It is now appreciated that multiple immune cell types traffic to adipose tissue in obesity, including macrophages and T cells. ${ }^{6}$ Accordingly, we measured expression of immune cell markers in visceral adipose tissue and found that $H$ felis infection specifically upregulated F4/80, a macrophage marker (figure 4D). There was no change in B cell, T cell or natural killer cell markers (figure 4D). Confirming this finding, we observed increased F4/80 cells in visceral adipose tissue by IHC (figure 4E). These data suggest that macrophages are the primary immune population affected by $H$ felis infection in adipose tissue of obese mice. In sum, $H$ felis infection increased macrophages in adipose tissue of obese mice, but not lean mice, which likely contributed to elevated serum proinflammatory cytokines IL-6 and leptin.

\section{$H$ felis infection augments monocyte/macrophage chemotaxis to adipose tissue}

Enhanced macrophage accumulation can occur through chemokine-based recruitment or proliferation of existing myeloid cells. ${ }^{38}$ To examine cell proliferation, we performed
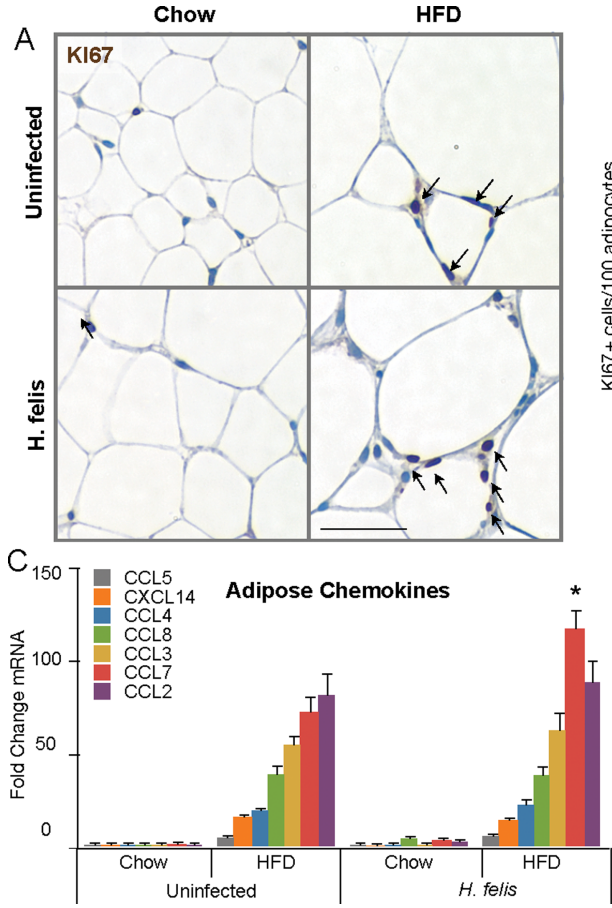

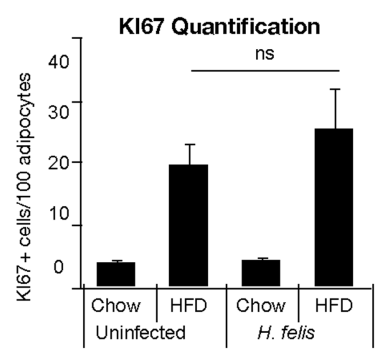

B

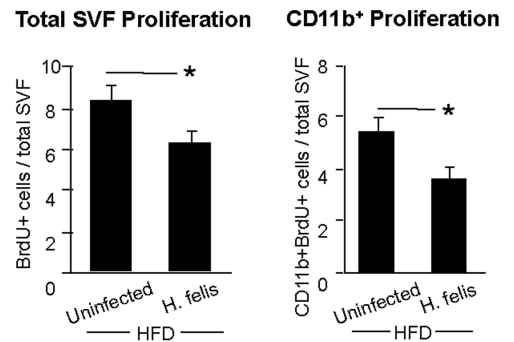

$\mathrm{D}$

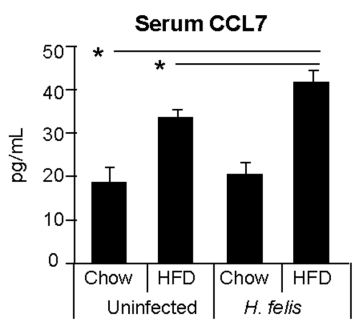

$F$

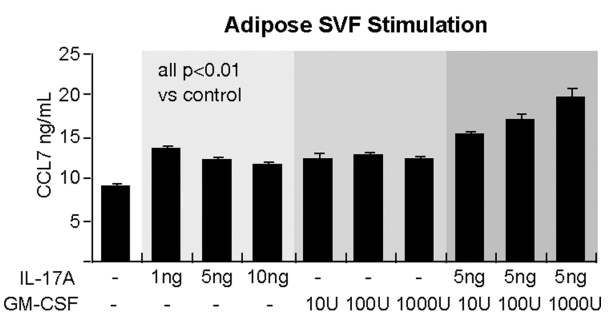

Figure 5 (A) Representative images of Ki67 immunohistochemistry in visceral adipose of high-fat diet (HFD) groups. Ki67 nuclei quantified, expressed as percentage of total adipocytes per high-power field. Minimum 3 representative fields quantified per mouse. Scale bar=50 $\mu \mathrm{m}$. (B) BrdU cells in visceral adipose Stromal vascular section (SVF), analysed by flow cytometry. Total SVF proliferation and total myeloid proliferation. Mice 2 months postinfection, sacrificed $18 \mathrm{~h}$ post-BrdU injection. (C) mRNA quantification of CCL2, 3, 4, 5, 7, 8 and CXCL14 in visceral adipose by qRT-PCR. (D) Serum CCL7 protein quantification by ELISA. (E) SVF cells isolated from uninfected/HFD visceral adipose, stimulated with recombinant IL-17A and/or GM-CSF for $72 \mathrm{~h}$. CCL7 in supernatant quantified by ELISA. N=3-4 wells/condition, one representative of three independent experiments shown. ${ }^{*} \mathrm{p}<0.05,{ }^{\#} \mathrm{p}<0.01,{ }^{\S} \mathrm{p}<0.001$. CCL, C-C motif ligand; $\mathrm{CXCL}$, Chemokine C-X-C motif ligand; GM-CSF, granulocyte macrophage-colony stimulating factor. 
Ki67 IHC on visceral adipose tissue and found no difference in Ki67 cells among study groups (figure 5A). To further investigate proliferation, BrdU cell populations were quantified by flow cytometry from whole visceral adipose tissue and SVF of BrdU-injected HFD study groups. Surprisingly, we saw a significant decrease in the percentage of total BrdU adipose tissue cells and CD11bBrdU myeloid cells in $H$ felis/HFD mice compared with uninfected/HFD mice (figure 5B), excluding proliferation as a major contributor to myeloid cell accumulation in $\mathrm{H}$ felis/ HFD adipose tissue.

To assess the role of adipose-derived chemokine recruitment of myeloid cells, we measured adipose tissue production of CCL- 2, 3, 4, 5, 7, 8 and CXCL14. Adipose chemokine production plays an important role in modulating obesity-associated inflammation. ${ }^{6}{ }^{8}$ Disruption of monocytic chemokine signalling pathways in obese mice can decrease adipose tissue macrophage infiltration and improve the adipose tissue inflammatory profile. ${ }^{39}$ Of the chemokines tested, only CCL7 was differentially expressed between infected and uninfected obese mice (figure 5C). Serum CCL7 was also significantly elevated in infected obese mice compared with uninfected controls (figure 5D). It is likely that elevated serum CCL7 protein in the $\mathrm{H}$ felis/ HFD mice is primarily a result of adipose-derived CCL7 production, as gastric tissue CCL7 mRNA was only slightly increased (see online supplementary figure S4C). Blood IMCs were not elevated after intravenous CCL7 injection, suggesting that, unlike CXCL1, CCL7 does not affect IMC mobilisation from the bone marrow to the blood (data not shown).

Last, we investigated if gastric-derived factors, including IL-17A and GM-CSF, were able to modulate adipose CCL7 production in obese mice. Although serum IL-17A concentrations were below our assay sensitivity for many mice, it could be detected in some infected mice. Serum GM-CSF levels in the four study groups showed a similar trend as seen in gastric tissue GM-CSF levels, with highest levels found in the $H$ felis/ HFD group (see online supplementary figure S4D). To assess modulation of CCL7 production, we stimulated visceral adipose SVF cells isolated from uninfected obese with recombinant IL-17A and/or GM-CSF for $72 \mathrm{~h}$. Stimulation with IL-17A or GM-CSF separately increased CCL7 production 1.5-fold. Stimulation with IL-17A and GM-CSF together increased CCL7 production 2-2.5-fold, in a dose-dependent manner (figure 5F). These data suggest that IL-17A and GM-CSF produced during $H$ felis infection may be able to stimulate CCL7 production in obese adipose tissue, increasing macrophage accumulation and subsequently, raising systemic inflammation.

\section{DISCUSSION}

In obese, compared with lean $H$ felis-infected mice, we observed severe gastritis and accelerated gastric carcinogenesis. These mice developed an enhanced protumorigenic gastric microenvironment, characterised by increased IMC infiltration and elevated $\mathrm{T}_{\mathrm{H}} 17$ response. Gastric epithelial STAT3 activity was also increased in these mice, in conjunction with heightened expression of prosurvival genes. At the same time, $H$ felis infection amplified adipose macrophage accumulation and proinflammatory cytokine production in visceral adipose tissue of obese, but not lean, mice.

Our results suggest that diet-induced obesity influences gastric carcinogenesis during $H$ felis infection through a proinflammatory positive feedback signalling loop between adipose and gastric tissues (figure 6). When adipose tissue becomes inflamed, it appears to become sensitised to circulating $H$ felis-associated proinflammatory signals. The resultant increase in adipose-

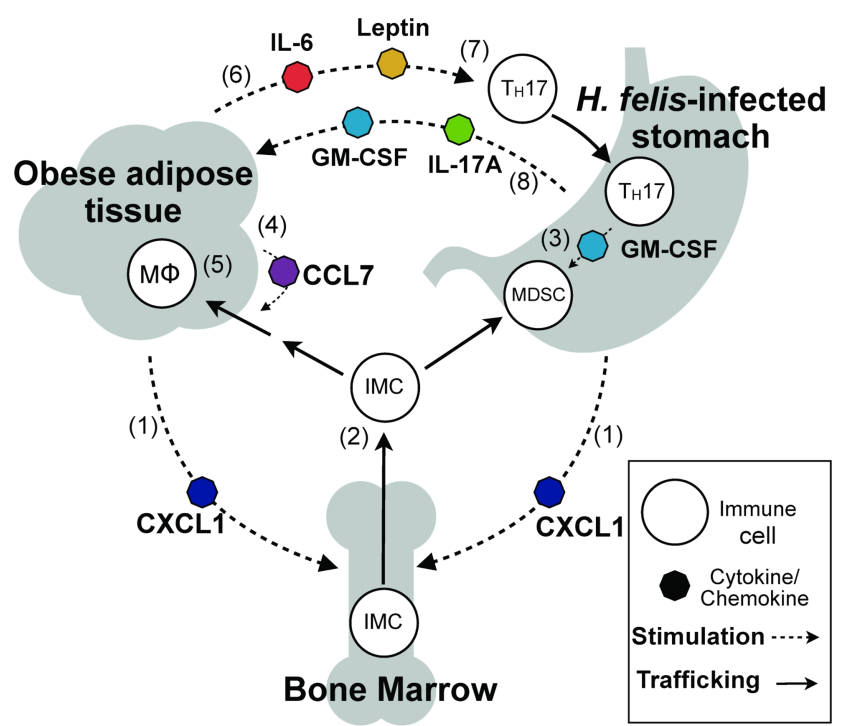

Figure 6 Schematic hypothesis of inflammatory cross-talk during Helicobacter felis infection and diet-induced obesity. CXCL1 produced by adipose and gastric tissues (1) elevates circulating bone marrow-derived immature myeloid cells (IMCs) (2). IMCs recruited to gastric tissue by $\mathrm{CXCL} 1$ encounter $\mathrm{T}_{\mathrm{H}} 17$-associated cytokines, including GM-CSF, and develop into myeloid-derived suppressor cells (3). Adipose CCL7 secretion increases IMC recruitment to adipose (4), resulting in accumulation of adipose tissue macrophages (MФ) (5). Inflamed adipose produces IL-6 and leptin (6), promoting $T_{H} 17$ differentiation (7). $T_{H} 17$-associated cytokines IL-17A and GM-CSF produced in the stomach signal to adipose (8) to increase CCL7 production and amplify adipose inflammation. $\mathrm{CCL}, \mathrm{C}-\mathrm{C}$ motif ligand; $\mathrm{CXCL}$, Chemokine $\mathrm{C}-\mathrm{X}-\mathrm{C}$ motif ligand; GM-CSF, granulocyte macrophage-colony stimulating factor.

derived serum factors modulates gastric IMC mobilisation (via CXCL1) and $\mathrm{T}_{\mathrm{H}} 17$ response (via IL-6 and leptin), both of which have been associated with a protumorigenic gastric microenvironment. $^{26} 2730$ Consequently, a positive feedback inflammatory signalling loop is established between adipose and gastric tissues during obesity and $H$ felis infection, accelerating the process of gastric carcinogenesis. It is important to note that, for this self-propagating signal to occur, the adipose tissue must already be inflamed, thereby 'priming' adipose tissue to respond to $H$ felis-associated cytokine signalling.

Based on our results, it is likely that obesity accelerates progression of gastric carcinogenesis during $H$ felis infection, in part, through induction of STAT3 signalling. In addition to STAT3-mediated promotion of cellular transformation in epithelial cells, ${ }^{31} 40$ STAT3 is also a master regulator of inflammatory gene expression and contributes to a cancer-promoting microenvironment. ${ }^{41}$ In obese infected mice, there was a dramatic increase in serum IL-6 and leptin levels which, at least partly, contributed to gastric STAT3 activation, despite unchanged gastric IL-6 and IL-11 expression. Specific STAT3 activation in the gastric epithelial cells of $H$ felis/HFD mice likely also involved localised inflammatory signalling factors, such as other interleukins and IFNs, known to activate STAT3. ${ }^{42}$ Consistent with our findings, IL- 6 has been shown to enhance tumour progression, ${ }^{43}$ and high serum IL-6 levels are negatively correlated with gastric cancer survival. ${ }^{35}$ Additionally, disruption of leptin signalling has been shown to reduce STAT3-mediated gastric hyperplasia. $^{44}$

Furthermore, we demonstrate that inflamed gastric and adipose tissues used CXCL1 to elevate circulating IMCs. 
CXCL1 is a known chemotactic factor for mature neutrophils, ${ }^{45}$ and studies with obese mice lacking the CXCL1 receptor CXCR2 have reduced neutrophil recruitment to adipose tissue. ${ }^{34}$ Furthermore, CXCL1 has also been shown to be upregulated in murine gastric preneoplasia, ${ }^{3345}$ suggesting a role for this chemokine in both gastric cancer and obesity. Here, we show that intravenous CXCL1 infusion leads to rapid, transient mobilisation of CD11bLy6G IMCs. Further studies are required to identify the specific activity of these IMCs, which have also been labelled polymorphonuclear MDSCs (PMN-MDSCs) ${ }^{46}$ in gastric tissue of $H$ felis-infected mice. Given our results, we suspect this IMC subset plays a pathogenic role in gastric carcinogenesis.

We also observed an increase in the gastric $\mathrm{T}_{\mathrm{H}} 17$ response, characterised by upregulated IL-17A and GM-CSF, upon addition of HFD in infected mice. Consistent with our findings, it has been recently suggested that IL-17A can foster gastrointestinal tumour development. ${ }^{25}$ Furthermore, the presence of IL-17A-producing cells in patients is associated with a worse prognosis for both gastric ${ }^{47}$ and colorectal cancers. ${ }^{48}$ Our data provides additional evidence that IL-17A is involved in inflammation-based gastrointestinal carcinogenesis and, furthermore, is sensitive to obesity-derived proinflammatory factors. We also demonstrate that IL-6 and leptin together can contribute to the initiation of a $\mathrm{T}_{\mathrm{H}} 17$ transcriptional profile in naive $\mathrm{T}$ cells, and speculate that leptin may be involved in $\mathrm{T}_{\mathrm{H}} 17$ differentiation and maintenance during obesity. We observed a marked and synergistic increase in GM-CSF expression in gastric tissue of obese, but not lean, infected mice. It has recently been proposed that GM-CSF production by $\mathrm{T}_{\mathrm{H}} 17$ cells is a critical mediator of $\mathrm{T}_{\mathrm{H}} 17$ pathogenicity. ${ }^{49}$ Independent of the $\mathrm{T}_{\mathrm{H}} 17$ response, GM-CSF has also been associated with accumulation of suppressive IMCs in multiple tumour models. ${ }^{16}{ }^{17}$ Future studies are needed to clarify the effect of leptin on GM-CSF production in $\mathrm{T}_{\mathrm{H}} 17$ cells and on $\mathrm{T}_{\mathrm{H}} 17$ pathogenicity.

Unexpectedly, $H$ felis infection increased adipose CCL7 chemokine production, macrophage recruitment and adipose inflammation in obese mice. $H$ felis infection did not alter inflammatory status of lean adipose tissue; thus, it appears that an already expanded, inflamed visceral adipose tissue is necessary for this process. It has been shown that knockout of CCL7 receptor, CCR2, reduces adipose inflammation, although the role of CCL7 in monocyte/macrophage recruitment to adipose tissue during obesity is still largely unknown. ${ }^{39}$ Intriguingly, CCL7 has previously been shown to increase after stimulation with IL-17A. ${ }^{23}$ In support of this finding, we show that IL-17A and/or GM-CSF, both of which were highly expressed in the $H$ felis-infected stomach, are able to stimulate CCL7 production from obese adipose tissue cells in vitro. Adipose-derived CCL7 may also have an adverse effect in the stomach, as high gastric CCL7 levels in gastric cancer patients has been correlated with more advanced tumour progression. ${ }^{50}$

Dysregulation of the gut microbiome by HFD and $H$ felis infection may also have increased systemic inflammation and contributed to the adipose or gastric pathology observed in $\mathrm{H}$ felis/HFD mice. Mice on HFD show altered gut microbiome composition and increased endotoxemia compared with mice on control diet. ${ }^{51}$ It would therefore be interesting to investigate if $\mathrm{H}$ felis infection and HFD together can further alter the gut microbiome, providing an additional mechanism through which $H$ felis and HFD work synergistically to accelerate gastric carcinogenesis.

In summary, we have identified a proinflammatory signalling mechanism between gastric and adipose tissues, coordinated by
Helicobacter infection and obesity. Over time, this proinflammatory milieu upregulated local and systemic proinflammatory factors and accelerated the process of gastric carcinogenesis. Obese inflamed adipose tissue, compared with lean adipose tissue, appears to have the unique ability to amplify $H$ felis-associated inflammation via a complex signalling loop involving IMC accumulation in blood and gastric tissue, $\mathrm{T}_{\mathrm{H}} 17$ response in gastric tissue and macrophage infiltration in adipose tissue. This signalling loop is only propagated in the presence of inflamed adipose tissue, as lean adipose tissue is not sensitised to respond to $H$ felis-associated inflammation. Epidemiological studies have demonstrated that $H$ pylori infection and obesity are independent risk factors for gastric cancer, ${ }^{45}$ although these risk factors may not coincide as often, given that obesity is not as common in countries where $H$ pylori infection is endemic. $^{52} 53$ Additionally, body mass index of individuals infected with $H$ pylori increases after eradication,${ }^{54}$ suggesting a possible inverse relationship between $H$ pylori infection and obesity. Nevertheless, our results clearly demonstrate that $H$ felis infection and HFD have a synergistic effect on gastric carcinogenesis, and may also provide insight into the mechanisms through which obesity increases risk of other solid cancers. It is possible that obesity can accelerate carcinogenesis in other organs through a similar immunomodulatory mechanism.

Acknowledgements The authors would like to thank Kelly Betz, Karan Nagar, Aliki Kosteli, Hye-Lim Noh, Ashley Whelan, Justin DeGrazia, Chintan Kapadia, Shanisha Gordon and Anthony Mitchell for technical assistance and animal care.

Contributors TCW, AWF: funding and study supervision. TCW, JGF, AWF, REE: study concept and design. REE, WS, SM, SR, YT: acquisition of data. REE, SR, CBW, RAF, JGF, AWF, WH, TCW: analysis and interpretation of data. SR, REE, CBW, JGF, AWF, TCW: drafting of manuscript.

Funding This research was supported by National Institute of Health grant 5R01CA093405-11 and Columbia University Diabetes Research Center grant P30DK063608.

Competing interests None.

Provenance and peer review Not commissioned; externally peer reviewed.

\section{REFERENCES}

1 Fox JG, Wang TC. Inflammation, atrophy, and gastric cancer. J Clin Invest 2007; 117:60-9.

2 Pinchuk IV, Morris KT, Nofchissey RA, et al. Stromal cells induce Th17 during Helicobacter pylori infection and in the gastric tumor microenvironment. PLOS ONE 2013;8:e53798

3 Coussens LM, Zitvogel L, Palucka AK. Neutralizing tumor-promoting chronic inflammation: a magic bullet? Science 2013;339:286-91.

4 Ahmed N, Sechi LA. Helicobacter pylori and gastroduodenal pathology: new threats of the old friend. Ann Clin Microbiol Antimicrob 2005;4:1.

5 Chung WK, Leibel RL. The links between obesity, leptin, and prostate cancer. Cancer J 2006;12:178-81.

6 Kanneganti TD, Dixit VD. Immunological complications of obesity. Nat Immunol 2012;13:707-12.

7 James BR, Tomanek-Chalkley A, Askeland EJ, et al. Diet-induced obesity alters dendritic cell function in the presence and absence of tumor growth. $J$ Immunol 2012;189:1311-21.

8 Ferrante AW. Obesity-induced inflammation: a metabolic dialogue in the language of inflammation. J Intern Med 2007;262:408-14.

9 Zuniga LA, Shen WJ, Joyce-Shaikh B, et al. IL-17 regulates adipogenesis, glucose homeostasis, and obesity. J Immunol 2010;185:6947-59.

10 Moon HS, Liu X, Nagel JM, et al. Salutary effects of adiponectin on colon cancer: in vivo and in vitro studies in mice. Gut 2013;62:561-70.

11 Zyromski NJ, Mathur A, Pitt HA, et al. Obesity potentiates the growth and dissemination of pancreatic cancer. Surgery 2009;146:258-63.

12 Khandekar MJ, Cohen P, Spiegelman BM. Molecular mechanisms of cance development in obesity. Nat Rev Cancer 2011;11:886-95.

13 Collins S, Martin TL, Surwit RS, et al. Genetic vulnerability to diet-induced obesity in the C57BL/6J mouse: physiological and molecular characteristics. Physiol Behav 2004:81:243-8. 
14 Wang TC, Dangler CA, Chen D, et al. Synergistic interaction between hypergastrinemia and Helicobacter infection in a mouse model of gastric cancer. Gastroenterology 2000;118:36-47.

15 Asfaha S, Dubeykovskiy AN, Tomita $\mathrm{H}$, et al. Mice that express human interleukin-8 have increased mobilization of immature myeloid cells, which exacerbates inflammation and accelerates colon carcinogenesis. Gastroenterology 2013;144:155-66.

16 Pylayeva-Gupta Y, Lee KE, Hajdu , et al. Oncogenic Kras-induced GM-CSF production promotes the development of pancreatic neoplasia. Cancer Cell 2012; 21:836-47.

17 Ostrand-Rosenberg S, Sinha P. Myeloid-derived suppressor cells: linking inflammation and cancer. J Immunol 2009;182:4499-506.

18 Wang L, Chang EWY, Wong SC, et al. Increased myeloid-derived suppressor cells in gastric cancer correlate with cancer stage and plasma S100A8/A9 proinflammatory proteins. J Immunol 2013;190:794-804

19 Serafini P, Borrello I, Bronte V. Myeloid suppressor cells in cancer: recruitment, phenotype, properties, and mechanisms of immune suppression. Semin Cancer Biol 2006;16:53-65.

20 Zhu B, Bando $Y$, Xiao S, et al. CD11b+Ly-6C(hi) suppressive monocytes in experimental autoimmune encephalomyelitis. J Immunol 2007;179:5228-37.

21 Shi Y, Liu XF, Zhuang Y, et al. Helicobacter pylori-induced Th17 responses modulate Th1 cell responses, benefit bacterial growth, and contribute to pathology in mice. J Immunol 2010:184:5121-9.

22 Fox JG, Beck P, Dangler CA, et al. Concurrent enteric helminth infection modulates inflammation and gastric immune responses and reduces helicobacter-induced gastric atrophy. Nat Med 2000;6:536-42.

23 Park $\mathrm{H}$, Li Z, Yang XO, et al. A distinct lineage of CD4 T cells regulates tissue inflammation by producing interleukin 17. Nat Immunol 2005;6:1133-41.

24 McGeachy MJ. GM-CSF: the secret weapon in the TH17 arsenal. Nat Immunol 2011:12:521-2.

25 Grivennikov S, Karin E, Terzic J, et al. IL-6 and STAT3 are required for survival of intestinal epithelial cells and development of colitis-associated cancer. Cancer Cell 2009;15:103-13

26 Ge Z, Feng Y, Muthupalani S, et al. Coinfection with Enterohepatic Helicobacter species can ameliorate or promote Helicobacter pylori-induced gastric pathology in C57BL/6 mice. Infect Immun 2011;79:3861-71.

27 Wang SS, Asfaha S, Okumura T, et al. Fibroblastic colony-forming unit bone marrow cells delay progression to gastric dysplasia in a helicobacter model of gastric tumorigenesis. Stem Cells 2009;27:2301-11.

28 Deng J, Liu Y, Yang $M$, et al. Leptin exacerbates collagen-induced arthritis via enhancement of Th17 cell response. Arthritis Rheum 2012;64:3564-73.

29 Shibata W, Ariyama H, Westphalen CB, et al. Stromal cell-derived factor-1 overexpression induces gastric dysplasia through expansion of stromal myofibroblasts and epithelial progenitors. Gut 2013;62:192-200.

30 Kennedy CL, Najdovska M, Jones GW, et al. The molecular pathogenesis of STAT3-driven gastric tumourigenesis in mice is independent of IL-17. J Pathol 2011:225:255-64.

31 Iliopoulos D, Jaeger SA, Hirsch HA, et al. STAT3 activation of miR-21 and miR-181b-1 via PTEN and CYLD are part of the epigenetic switch linking inflammation to cancer. Mol Cell 2010;39:493-506.

32 Tu S, Bhagat G, Cui G, et al. Overexpression of interleukin-1 beta induces gastric inflammation and cancer and mobilizes myeloid-derived suppressor cells in mice. Cancer Cell 2008;14:408-19.

33 Okumura $\mathrm{T}$, Ericksen RE, Takaishi $\mathrm{S}$, et al. K-ras mutation targeted to gastric tissue progenitor cells results in chronic inflammation, an altered microenvironment, and progression to intraepithelial neoplasia. Cancer Res 2010;70:8435-45.
34 Neels JG, Badeanlou L, Hester KD, et al. Keratinocyte-derived chemokine in obesity: expression, regulation, and role in adipose macrophage infiltration and glucose homeostasis. J Biol Chem 2009:284:20692-98.

35 Jackson CB, Judd LM, Menheniott TR, et al. Augmented gp130-mediated cytokine signaling accompanies human gastric cancer progression. J Pathol 2007;213:140-51.

36 Hirota $\mathrm{K}$, Yoshitomi $\mathrm{H}$, Hashimoto $\mathrm{M}$, et al. Preferential recruitment of CCR6-expressing Th17 cells to inflamed joints via CCL20 in rheumatoid arthritis and its animal model. J Exp Med 2007;204:2803-12

37 Teixeira LG, Leonel AJ, Aguilar EC, et al. The combination of high-fat diet-induced obesity and chronic ulcerative colitis reciprocally exacerbates adipose tissue and colon inflammation. Lipids Health Dis 2011;10:204-19.

38 Osborn 0, Olefsky JM. The cellular and signaling networks linking the immune system and metabolism in disease. Nat Med 2012;18:363-74.

39 Weisberg $\mathrm{S}$, Hunter D, Huber R, et al. CCR2 modulates inflammatory and metabolic effects of high-fat feeding. J Clin Invest 2006;116:115-24.

40 Bromberg JF, Wrzeszczynska MH, Devgan G, et al. STAT3 as an oncogene. Cell 1999;98:295-303.

$41 \mathrm{Yu}$ H, Pardoll D, Jove R. STATs in cancer inflammation and immunity: a leading role for STAT3. Nat Rev Cancer 2009;9:798-809.

42 Grivennikov SI, Karin M. Dangerous liaisons: STAT3 and NF-kB collaboration and crosstalk in cancer. Cytokine Growth Factor Rev 2010;21:11-19.

43 Grivennikov S, Greten FR, Karin M. Immunity, inflammation, and cancer. Cell 2010;140:883-99.

44 Inagaki-Ohara K, Mayuzumi $\mathrm{H}$, Kato $\mathrm{S}$, et al. Enhancement of leptin receptor signaling by SOCS3 deficiency induces development of gastric tumors in mice. Oncogene. Published Online First: 26 November 2012. doi:10.1038/onc.2012.540

45 Sheh A, Ge Z, Parry NMA , et al 17 -estradiol and tamoxifen prevent gastric cancer by modulating leukocyte recruitment and oncogenic pathways in Helicobacter pylori-Infected INS-GAS male mice. Cancer Prev Res 2011;4:1426-35.

46 Youn J, Kumar V, Collazo M. Epigenetic silencing of retinoblastoma gene regulates pathogenic differentiation of myeloid cells in cancer. Nat Immunol 2013;14:211-20.

47 Zhuang Y, Peng LS, Zhao YL, et al. CD8(+) T cells that produce interleukin-17 regulate myeloid-derived suppressor cells and are associated with survival time of patients with gastric cancer. Gastroenterology 2012;143:951-62.

48 Tosolini M, Kirilovsky A, Mlecnik B, et al. Clinical impact of different classes of infiltrating t cytotoxic and helper cells (Th1, Th2, Treg, Th17) in patients with colorectal cancer. Cancer Res 2011;71:1263-71.

49 El-Behi M, Ciric B, Dai H, et al. The encephalitogenicity of TH17 cells is dependent on IL-1- and IL-23-induced production of the cytokine GM-CSF. Nat Immunol 2011;12:568-75

50 Hwang T, Lee L, Wang C, et al. CCL7 and CCL21 overexpression in gastric cancer is associated with lymph node metastasis and poor prognosis. World I Gastroenterol 2012:18:1249-56

51 Frazier TH, DiBaise JK, McClain CJ. Gut microbiota, intestinal permeability, obesity-induced inflammation, and liver injury. J Parenter Enteral Nutr 2011:35:14S-20S

52 Mitchell HM. Epidemiology of infection. In: Mobley HLT, Mendz GL, Hazell SL, eds. Helicobacter pylori: physiology and genetics. Washington, DC: ASM Press 2001:7-18

53 Kelly T, Yang W, Chen CS, et al. Global burden of obesity in 2005 and projections to 2030. Int J Obes 2008;32:1431-7.

54 Lane JA, Murray LJ, Harvey IM, et al. Randomised clinical trial: Helicobacter pylori eradication is associated with a significantly increased body mass index in a placebo-controlled study. Alim Pharm Ther 2011:33:922-9. 